\title{
Multi-channel Feedarray Reflector Antenna Based Radar Concept for HRWS SAR Imaging
}

\author{
Javier del Castillo, Lara Orgaz and Quiterio \\ Garcia \\ Airbus Defence and Space \\ Avd. Aragon 404, Madrid, Spain \\ javier.delcastillo@airbus.com \\ lara.orgaz@airbus.com \\ quiterio.garcia@airbus.com
}

\author{
Nafsika Memeletzoglou \\ Dpt. Signal Theory and Communications \\ Universidad Carlos III of Madrid \\ Leganés, Spain \\ nausika@tsc.uc3m.es \\ Carlos Biurrun-Quel and Carlos del-Río \\ Institute of Smart Cities/Antenna Group \\ Universidad Pública de Navarra \\ Navarra, Spain \\ carlos@unavarra.es
}

Giovanni Toso and Ernesto Imbembo Radio Frequency Payloads \& Technology Division

European Space Agency, ESA/ESTEC

Keplerlaan 1, Noordwijk, The Netherlands giovanni.toso@esa.int ernesto.imbembo@esa.int

\begin{abstract}
Large deployable reflector based multi-channel antenna systems are a key component of next generation SAR missions as they allow implementing multi-beam imaging capabilities and provide an efficient way to overcome the classical SAR resolution-coverage trade-off, covering wide swaths with high azimuth resolution. The presented work shows the design of a multichannel L-band SAR system and antenna architecture based on different feeders techniques which is suitable for next generation L-band SAR missions and capable to map $400 \mathrm{~km}$ ground swath with 5 meter spatial resolution. The paper describes a preliminary design and architecture of the SAR system and antenna where different design aspects including beamforming concepts and feedarray implementation techniques have been identified and assessed in view of achieving the required performances while reducing the overall complexity of the solution.
\end{abstract}

\section{Keywords - Synthetic Aperture Radar, digital beamforming}

\section{INTRODUCTION}

One of the main limitations of state-of-the-art SAR is the impossibility to illuminate a wide swath while providing a high resolution at the same time due to conflicting requirements on the PRF. SAR systems are therefore limited in that a wide swath can only be imaged at the expense of a degraded azimuth resolution.

To overcome classical SAR limits digital beam forming SAR (DBF-SAR) architectures based on multiple receiver channels have been proposed in the recent years as the base for future SAR systems [1]. Multiple simultaneous elevation Rx beams allow extending the imaged swath beyond the limit of range ambiguities by acquiring echo signals from different portions of the desired swath which are received simultaneously. These simultaneous echoes can be properly separated by the usage of the SCan On REceive technique (SCORE)[2] and the principle that the desired signal and the ambiguities come from different directions. If the antenna system architecture allows several $\mathrm{Rx}$ beams to be formed simultaneously, multi-beam SCORE will enable following the radar ground returns from different subswaths simultaneously [3]. The main drawback of this operation concept is the emergence of blind ranges between subswaths which prevents the mapping of a wide swath without gaps produced due to the radar pulse transmission instances [4-5]. If the PRI is uniform, blind ranges remain unchanged along azimuth. Staggered SAR technique allows alleviating the presence of blind ranges in a system with multiple elevation beams and thus allows imaging a wide continuous swath [6]. A staggered SAR with multiple azimuth channels could even allow a further increase of the azimuth resolution, while keeping the wide continuous swath [7]. The inclusion of multiple azimuth channels allows a gain in the azimuth sampling rate supporting an improved azimuth resolution without the need to increase the PRF of the radar.

From radar system technology point of view, an efficient way to implement elevation multi-beam Staggered SAR with multiple azimuth channels is to design a large reflector based antenna illuminated by a feedarray organized in overlapped subarrays, supporting novel DBF-SAR architectures which exploit multiple and simultaneous available beams in both azimuth and range/elevation directions.

This paper describes different antenna beamforming concepts, presents promising feedarray implementation techniques and provides a preliminary radar system architecture design to achieve the required SAR reference mission performances specified on Table I.

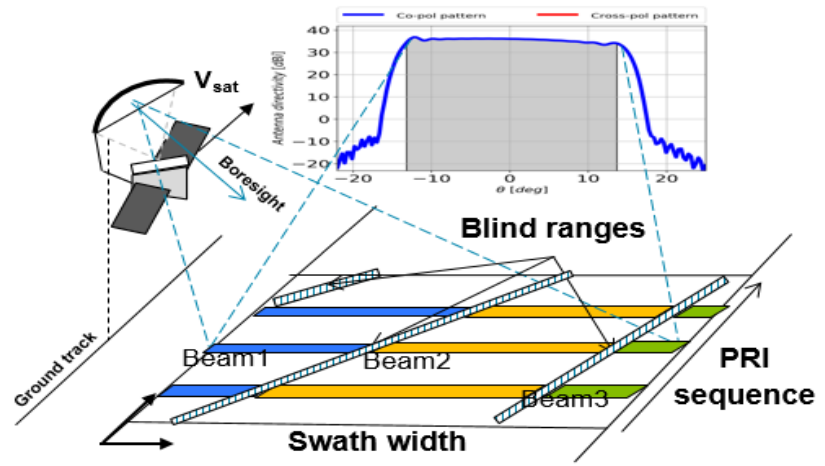

Fig. 1. Multi-beam SCORE stagered SAR stripmap operation mode

TABLE I. TARGET SAR MISSION REQUIREMENTS

\begin{tabular}{|ll|}
\hline \multicolumn{2}{|c|}{ SAR requirements } \\
\hline \multicolumn{1}{|c|}{ Parameter } & \multicolumn{1}{c|}{ Value } \\
\hline Swath width & $400 \mathrm{~km}$ \\
\hline Centre frequency & $1.2575 \mathrm{GHz}$ \\
\hline Resolutuon [range $\mathrm{x}$ azimuth] & $5 \mathrm{~m} \times 5 \mathrm{~m}$ \\
\hline NESZ & $-28 \mathrm{~dB}$ \\
\hline TAR & $-23 \mathrm{~dB}$ \\
\hline
\end{tabular}




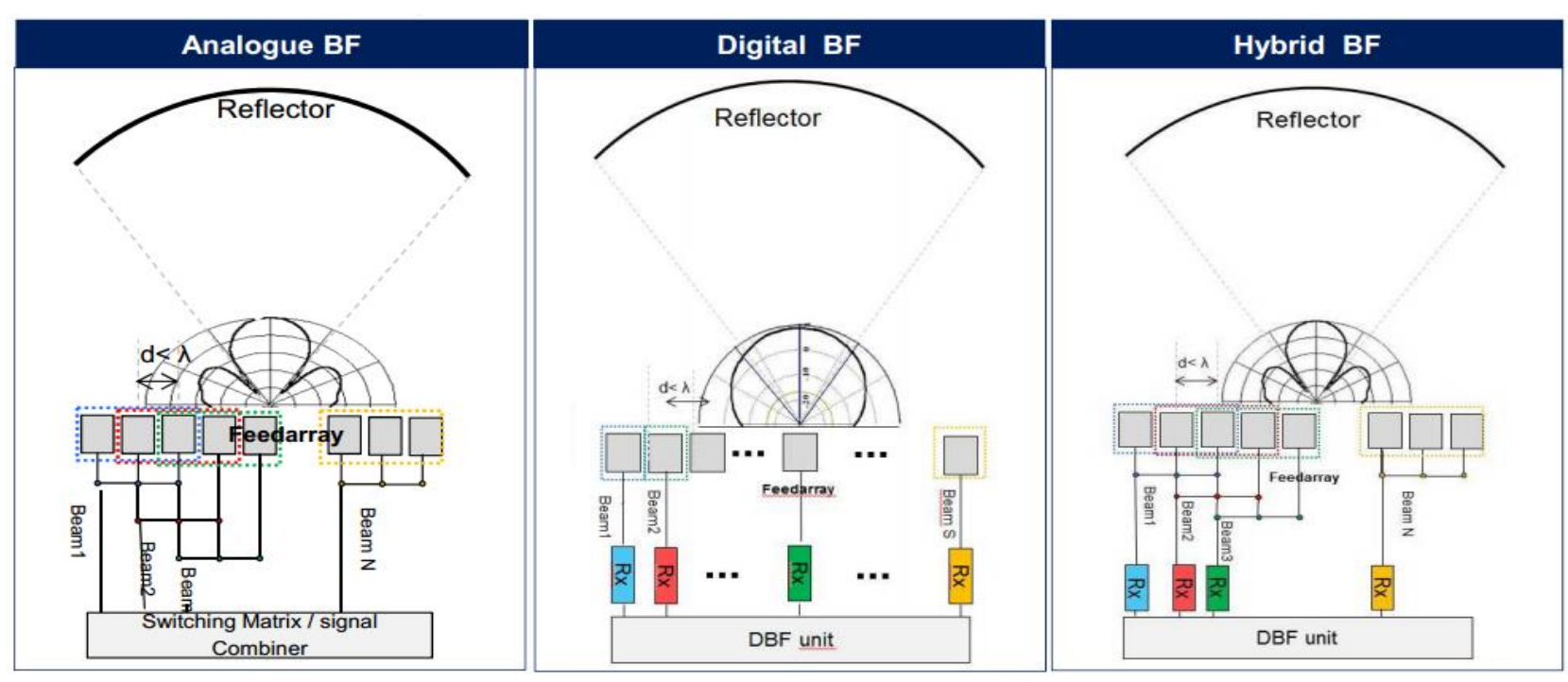

Fig. 2. Rx Antenna pattern Beamforming realizations of ScORe technique

\section{RX BEAMFORMING CONCEPTS}

Different beamforming concepts have been analysed to identify the most suitable approach to achieve the required radar performances. Digital, analogue and hybrid analogue/digital beamforming have been traded-off.

\section{A. Analogue Beamforming}

Individual antenna apertures are combined into feed clusters being some of the apertures shared between adjacent clusters. As it will be latter discussed, the combination can be achieved either by adopting beamforming networks or in a radiative way (i.e. exploiting the presence of parasitic structures placed in front of the feed apertures). The result is an effective illumination of the reflector by each equivalent feed element (cluster) and the tight separation between consecutive feed elements that can be achieved (being a fraction of wavelength).

One critical aspect of this approach is that the equivalent feed element area may be increased as far as the cluster positions goes away of the reflector focal point. This is due to the inherent defocusing of the secondary pattern that might be compensated in order to optimise the illumination from the feed array cluster. Finally, the available secondary pattern beams are fixed to a discrete number with different pointing, being the implementation of SCORE limited to a consecutive beam switching which reduces the NESZ improvement and prevents the implementation of adaptive beamforming algorithms.

\section{B. Digital beamforming}

Given a fixed size of the feedarray, two approaches can be considered. On one hand, a digital beamforming unit can be fed by multiple signals each one obtained from the combinations of $\mathrm{P}$ individual apertures into feed clusters. Each cluster properly illuminates the reflector surface but with a high interspacing between them. On the other hand, one can feed the digital beamforming unit with the signals from individual apertures that do not properly illuminate the reflector but have achieving a separation between apertures as small as the one achieved in the analogue beamfoming solution.

In both approaches the DBF unit allows synthetizing a huge amount of beams enabling not only SCORE but also adaptive beamforming algorithms (i.e. MVDR, LCMV, etc). However, in the first configuration the number of available digital channels is reduced thus achieving a smaller DBF gain but a better spill over performance per beam. The second configuration provides a worse spill over performance per beam but a greater DBF gain due to a higher secondary beams overlap.

\section{Hybrid digital and analogue beamforming}

A solution which combines both previous discussed approaches can be also considered. As in the analogue approach, individual apertures are combined in an analogue way into clusters being some of the apertures shared between adjacent clusters. The signals from the multiple clusters feed a digital beamforming unit which enables a further pattern improvement. One can benefit of this improvement to slightly relax the requirements of the analogue beamforming solution. For instance the number of combined apertures per cluster can be fixed to a smaller value even for the clusters located at the edges of the feed array assuming that certain level of pattern defocusing can be compensated by latter digital beamforming processing algorithms.

\section{SAR ANTENNA BASELINE CONCEPT}

The proposed SAR antenna architecture is based on a feed-array reflector antenna with a hybrid digital/analogue beamforming approach that enables a wide Tx beam pattern in elevation in combination with multiple and simultaneous narrow high gain Rx beams. Each Rx beam is generated by combining only a small subset of equivalent feed elements beams in concordance with the direction of the echo signal to be received. The synthetized receive beam performs the so called SCORE by digitally combining the inputs from multiple parallel received subsets of equivalent feed elements. 
Figure 3 depicts the instrument architecture for two different feedarray overlapping concepts. Transmission path components are the yellow coloured ones (HPAs, phase shifters polarization switches and circulators). Reception path components (LNAs, filters, ADCs and DBF units) are coloured in red for $\mathrm{V}$ polarization and blue for $\mathrm{H}$ polarization. The units in charge of enabling the apertures overlap is labelled as A-BFN and EBG depending on overlapping concept. It shall be noted that for simplicity, overlapping has been represented only in one dimension (elevation) while it might be implemented in two dimensions (elevation and azimuth) if required. Each of the antenna aperture symbols on the left sides represents an equivalent feed element that will then become and available system elevation channel.

The Tx beamforming strategy will be purely analogue. The phase control on transmit has been included to optimize the transmit characteristic of the radiation pattern if needed. The transmit radar signal will be routed first via analogue azimuth beamforming network and later split in elevation inside each azimuth column by an elevation BFN. The signal is then amplified by distributed HPAs having as many units as equivalent feed elements.

On receive the echo signal acquired by each equivalent feed element (apertures cluster in case of BFN approach or individual apertures in the EBG approach) is low noise amplified and conditioned. The $\mathrm{N}$ received channels are digitised and downconverted thus having $\mathrm{N}$ available analogue beams partially overlapped on ground. Finally, the elevation digital beamforming is performed before data is compressed, formatted and recorded in the SSMM. In the digital beamforming unit (DBFU) the $N$ complex data streams will be used to synthetize a simultaneous number of beams (in our case 5) each one performing SCORE to cover a portion of the complete $400 \mathrm{~km}$ imaged swath. In addition, DBF technique provides the system with adaptive beamforming capability, which enables the implementation of complex beamforming techniques in reception to improve ambiguous returns rejection.

The SAR antenna main parameters (Reflector and feedarray) are summarized in Table II.

TABLE II. SAR ANTENNA DESIGN PARAMETERS

\begin{tabular}{|l|l|l|l|}
\hline \multicolumn{2}{|c|}{ Reflector } & \multicolumn{2}{c|}{ Feed array } \\
\hline \multicolumn{1}{|c|}{ Parameter } & \multicolumn{1}{c|}{ Value } & \multicolumn{1}{c|}{ Parameter } & \multicolumn{1}{c|}{ Value } \\
\hline Diameter $\mathrm{x}$ & $15 \mathrm{~m}$ & Spacing (x /y) & $\sim 1 \lambda / 1 \lambda$ \\
\hline Diameter y & $15 \mathrm{~m}$ & Beamforming losses & $2 \mathrm{~dB}$ \\
\hline Focal length & $13.5 \mathrm{~m}$ & Feed tilt & $41^{\circ}$ \\
\hline Offset & $10.5 \mathrm{~m}$ & El elements/channels & $34 / 34$ \\
\hline Boresight angle & $36.5^{\circ}$ & Az elemets/channels & $3 / 3$ \\
\hline
\end{tabular}

The reflector diameter is $15 \mathrm{~m}$; focal length is $13.5 \mathrm{~m}$ and offset $10.5 \mathrm{~m}$. The offset and the focal length have been chosen to minimize defocusing effects on the secondary patterns. Offset is optimized to avoid reflector field of view blockage by the feedarray. The boresight angle (off nadir angle) of the antenna is $36.5^{\circ}$.

The feedarray is formed by 34 elements in elevation and 3 in azimuth, spaced in the range of $1 \lambda$ on each dimension.
The feed elements tilt angle is derived geometrically from previous values in order to properly illuminate the reflector surface. During radar transmision pulse gates, the first 28 elevation rows starting from the top side of the feedarray are assumed to be active. On receive; SCORE beamforming will be applied at each slant range combining the signal from adjacent overlapped secondary patterns.

Analogue beamforming losses are $2 \mathrm{~dB}$ while $\mathrm{Rx}$ noise figure is $4 \mathrm{~dB}$.
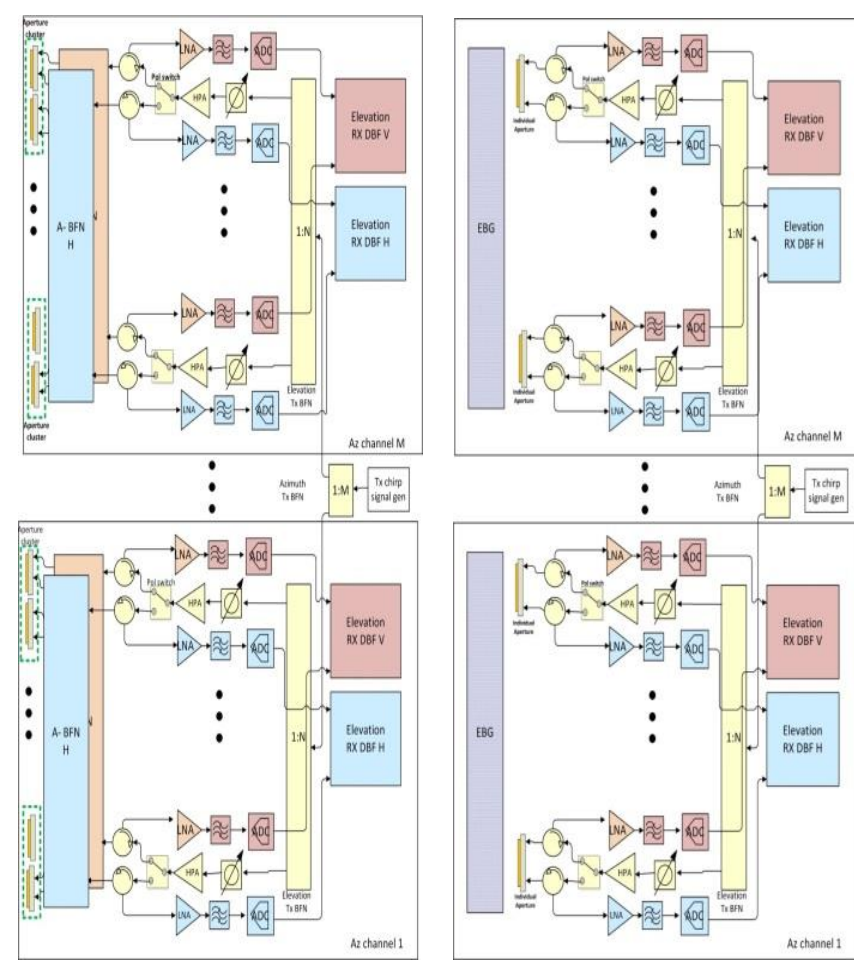

Fig. 3. SAR Antenna feedarray implamentation concepts. Left) CORPSBFN technique, Right) Meta-material surface Leaky wave technique

\section{SUBARRAY OVERLAPPING TECHNIQUES}

The need for highly directive overlapped beams covering a large ground area and generated from different feed elements in an array configuration with a tight separation between feeds leads to inefficient illumination of the reflector and insufficient performance in terms of achieved directivity and thus required transmit power.

To overcome this limits it is highly recommended to implement overlapped subarray technique to increase the aperture size of the feeds and thus achieve efficient illumination of the reflectors enabling low spillover losses with the possibility of creating contiguous overlapped beams with a low required cross-over level.

Two solutions can be adopted to achieve the overlap between radiators eiher by using waveguides for signal distribution/combination or adopting a radiative way combination approach. Both technqies are described below as CORPs-BFN and Leaky wave antenna techniques.

\section{A. CORPS-BFN Technique}

Coherently Radiating Periodic Structures - Beam Forming Network (CORPS-BFN) were proposed in the last 
decade [8-9] and present interesting properties for feeding antenna arrays. The proposed configuration for this case, would be a single layer of a hexagonal CORPS-BFN, defining sub-arrays of three small radiators equally illuminated from the common input. Since the beam forming is shared and common to all the feeding array, these three small radiators also receive contributions from the neighboring inputs, overlapping the effective radiating areas of the subarrays.

The hexagonal CORPS-BFN is based on a Gysel power divider/combiner of three outputs, implemented in a planar technology with a coaxial input port. This power combiner features the required return loss and isolation and provides a higher power-handling capabilities than the Wilkinson combiner [10]. In this case, the Gysel cell was implemented on a suspended stripline technology, Figure 4. The substrate is then encapsulated and suspended in between two metallic enclosures that include an air-filled channel, forming the suspended stripline. The power combiner was simulated in HFSS and Figure 5 presents its S-Parameters. It can be observed that both isolation and return loss requirements are below $-25 \mathrm{~dB}$, while very low insertion loss is achieved (ideal S21 value is around $-4.77 \mathrm{~dB}$, corresponding with an amplitude of $1 / \sqrt{ } 3$, and the simulated value lays around $4.9 \mathrm{~dB})$.

The final hexagonal CORPS-BFN is constructed interleaving Gysel cells dividing and combining, physically flipping the structure alternatively within a triangular lattice, in a way that all input coaxial ports are in one of the sides of the structure and all the output coaxial ports in other side.

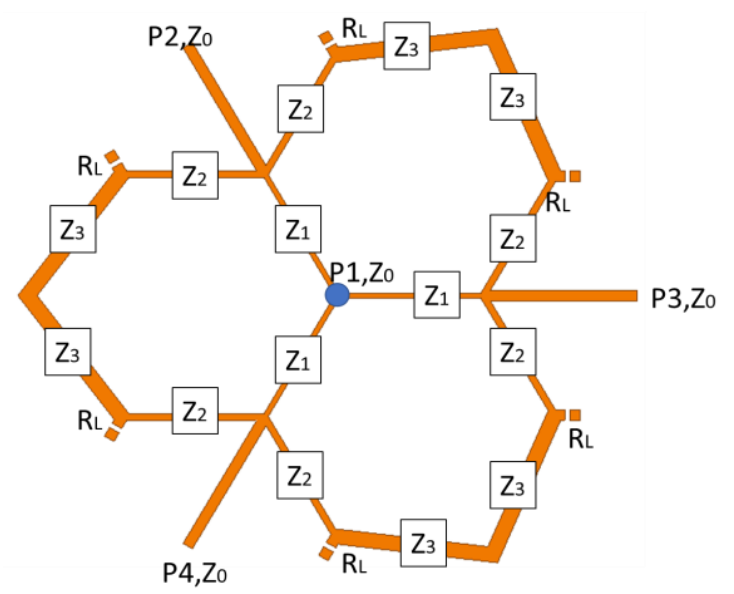

Fig. 4 Sketch of the proposed 4-port Gysel power combiner/divider, highlighting the different impedance section. Every sections consist of a quarter-wavelength transformer.

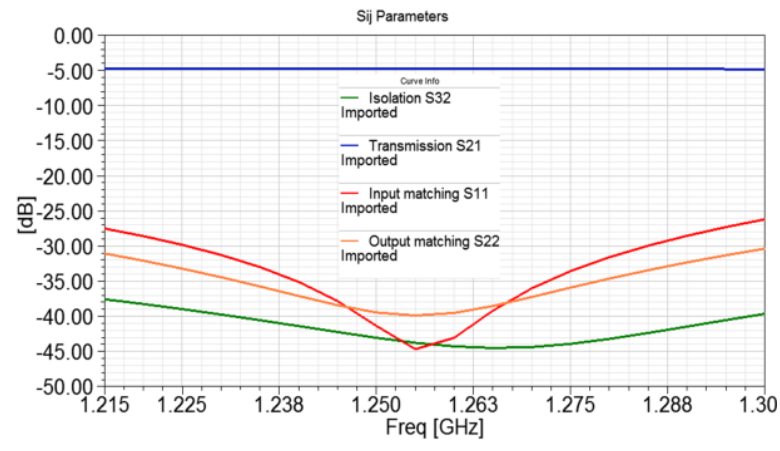

Fig. 5 S-Parameters of the proposed Gysel cell computed by HFSS.

\section{B. Meta-material surface based Leaky wave technique}

An alternative lossless approach in order to provide a dense beam grid with low spillover losses is the use of superlayers (made with dielectrics or with metasurfaces) on top of the antenna radiating apertures. This super-layer causes a guided leaky wave between the antenna plane and the surface plane that increases the electrical aperture of the original element [11]. The advantage of the solution based on leaky wave antennas is the simplicity and the ability to obtain the desired performance in terms of equivalent feed element directivity, cross polarization level and low losses. The main weak points of this tehcnique are the reduced bandwidth (frequency sensitivity) and high mutual coupling between radaiting apertures.

The designed super-layer controls the size of the illuminated aperture and consequently the directivity of each antenna. A more reflective super-layer will produce a more directive radiation pattern, but at the same time, the coupling with the neighboring antenna will increase. This coupling will compromise the performance of this solution due to its effect on the embedded radiation pattern.

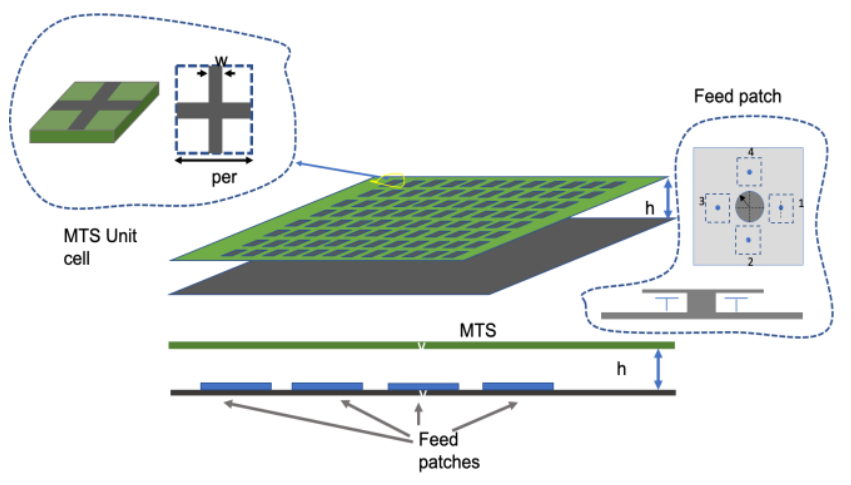

Fig.6 Description of the solution based on the use of leaky wave antennas with a MTS layer

As depicted on Figure 6, the proposed solution uses a grid-type metasurface equivalent to a dielectric layer on top of an array of dual polarization patch antennas with differential feed probe schemes in order to guarantee the mentioned low cross-polarization and a reltive wide frequency bandwidth of operation at L-band. 


\section{SAR SYSTEM PERFORMANCES}

Table III summarizes the main geometry, radar and SAR operation parameters wich have been derived in order to map a $400 \mathrm{~km}$ swath with $5 \mathrm{~m}$ spatial resolution.

TABLE III. SAR REQUIREMENTS AND RADAR PARAMETERS

\begin{tabular}{|c|c|c|c|c|c|}
\hline \multicolumn{2}{|c|}{ Geometry } & \multicolumn{2}{|c|}{ Radar } & \multicolumn{2}{|c|}{ Operation } \\
\hline Parameter & Value & Parameter & Value & Parameter & Value \\
\hline $\begin{array}{l}\text { Min look } \\
\text { angle }\end{array}$ & $23^{\circ}$ & $\begin{array}{l}\text { Average Tx } \\
\text { power }\end{array}$ & 270W & Mode & Stripmap \\
\hline $\begin{array}{l}\text { Max look } \\
\text { angle }\end{array}$ & $45^{\circ}$ & Noise figure & $4 \mathrm{~dB}$ & PRF & Staggered \\
\hline Orbit height & $\begin{array}{l}720 \\
\mathrm{~km}\end{array}$ & $\begin{array}{l}\text { System } \\
\text { losses }\end{array}$ & $2 \mathrm{~dB}$ & PRF mean & $2200 \mathrm{~Hz}$ \\
\hline $\begin{array}{l}\text { Swath } \\
\text { width }\end{array}$ & $\begin{array}{l}400 \\
\mathrm{Km}\end{array}$ & $\begin{array}{l}\text { Tx duty } \\
\text { cycle }\end{array}$ & $5 \%$ & $\begin{array}{c}\text { SCORE } \\
\text { beams }\end{array}$ & 5 \\
\hline Resolution & $5 \mathrm{~m}$ & Tx BW & $\begin{array}{l}85 \\
\mathrm{MHz}\end{array}$ & $\begin{array}{l}\text { Process } \\
\text { window }\end{array}$ & $\begin{array}{l}\text { Haming } \\
0.65\end{array}$ \\
\hline
\end{tabular}

The acquisition look angle is limited to the range from $23^{\circ}$ to $45^{\circ}$. This provides a ground range swath width of 400 $\mathrm{km}$ for an orbit height of $720 \mathrm{Km}$. The radar system operates following a staggered SAR timming in a Stripmap acquisition mode, where the pulse repetition interval (PRI) is changed from pulse to pulse. With this method the blind ranges are distributed in a deterministic way over range and a gapless SAR acquisition can be created by interpolating the SAR raw data. The actual PRF sequence is shown in Figure 7 , the average PRF is $2200 \mathrm{~Hz}$ and the number of requried simultaneous SCORE beams is 5 .

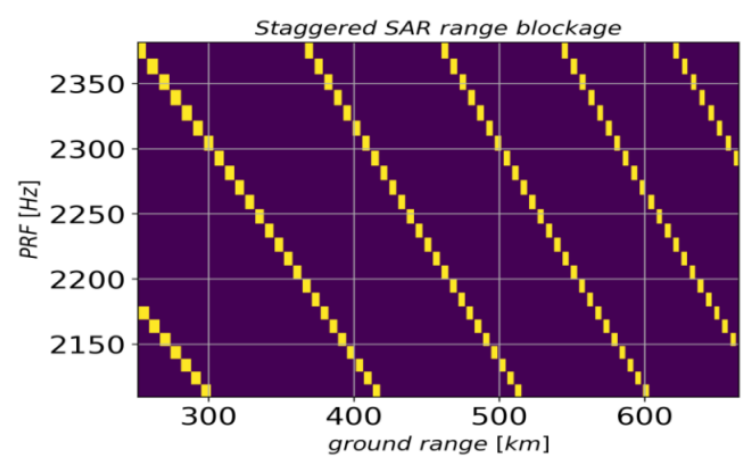

Fig. 7. Staggered operation PRF sequence optimized showing the corresponding blocked ranges in yellow and how they desplace in range accordingly due to the PRF variation

The plot in Figure 8 shows the analogue secondary patterns of the beams available for later digital beamforming combination for the central feed array column. Combining the first 28 beams in transmission allows achieving wide elevation pattern (black colour dashed curve) with good enough directivity and therefore meeting the sensitivity in the whole swath. The different colours solid lines represent the $34 \mathrm{Rx}$ available elevation beams. It might give the impression that there is an abundance of beams, especially at near and far range where beamwidth start to increase due to defocusing effect. These additional beams will enable to compensate the defocusing effect induced by the large scan angle by combining more secondary pattern contributions in the digital beamforming process.

Regarding the digital Rx beamforming algorithm, it has been implemented by multiplying each elevation channel by a complex weight in a time-variant manner (depending on the signal direction to be maximized) and summing up the signals. In this example the amount of combined analogue channels is limited to the ones providing pattern directivity at the desired signal direction of arrival above a $-20 \mathrm{~dB}$ threshold below the maximum beam directivity at the desired signal direction.

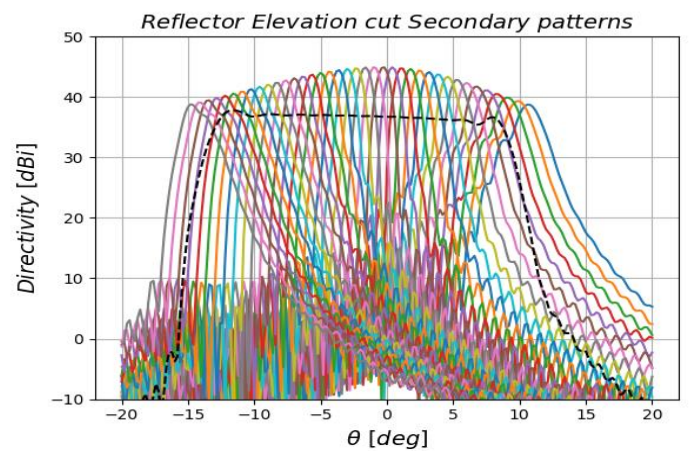

Fig. 8. Tx (dashed balck) and Rx (solid colours) Reflector elevation cut Secondary Patterns

The plots in Figure 9 depict an example of the Rx digital weigths needed to point the synthetized beam to near range. The botton plot shows the Rx synthetized beam corresponding to near range swath edge with range ambiguous positions marked as black dots. It shall be noticed that achieved directivity increase due to DBF implementation, in special at the limits of the scanned area, as a result of sharping the beam pattern shape compensating the observed analogue secondary pattern defocusing. This is a key aspect to perform SCORE technique and achieve the stringent NESZ requirement in such a wide swath.
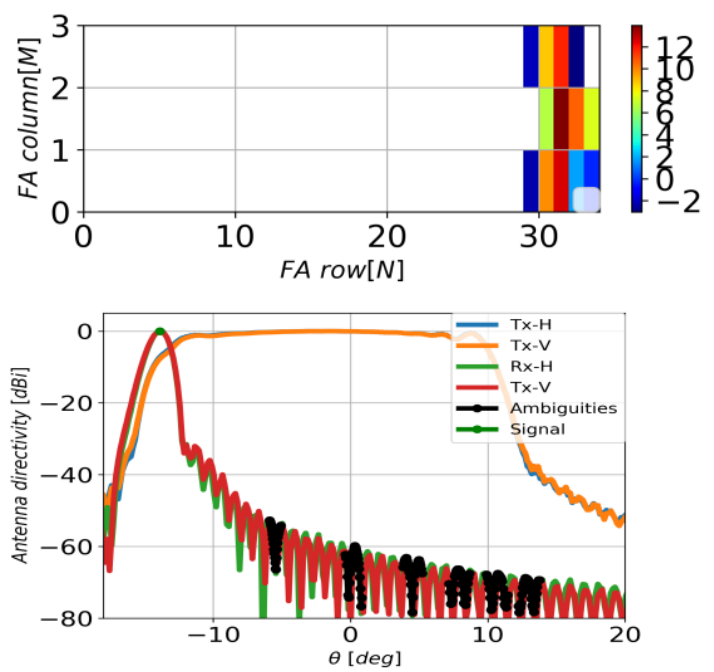

Fig 9. Top) Rx digital weights amplitude to synthetize a near range pointed Rx pattern. Botton) Normalized Tx[blue and orange] and Rx [green and red] beam patterns with range ambiguous regions as black dots over $\mathrm{Rx}$ beam.

Figure 10 provides a preliminary assessment of the SAR system performances in dual polarization mode over the 400 $\mathrm{km}$ swath width. The NESZ is better than $-28 \mathrm{~dB}$ for the average transmitted power of $270 \mathrm{~W}$. The RASR is better than $-23.0 \mathrm{~dB}$. Finally, the AASR is better than $-24 \mathrm{~dB}$ and the resolution is better than $5 \mathrm{~m}$. For range ambiguities 
computation the Ulaby L-band backscattering model [12] for shrubs has been considered ( $50 \%$ occurrence $\sigma 0$ values).
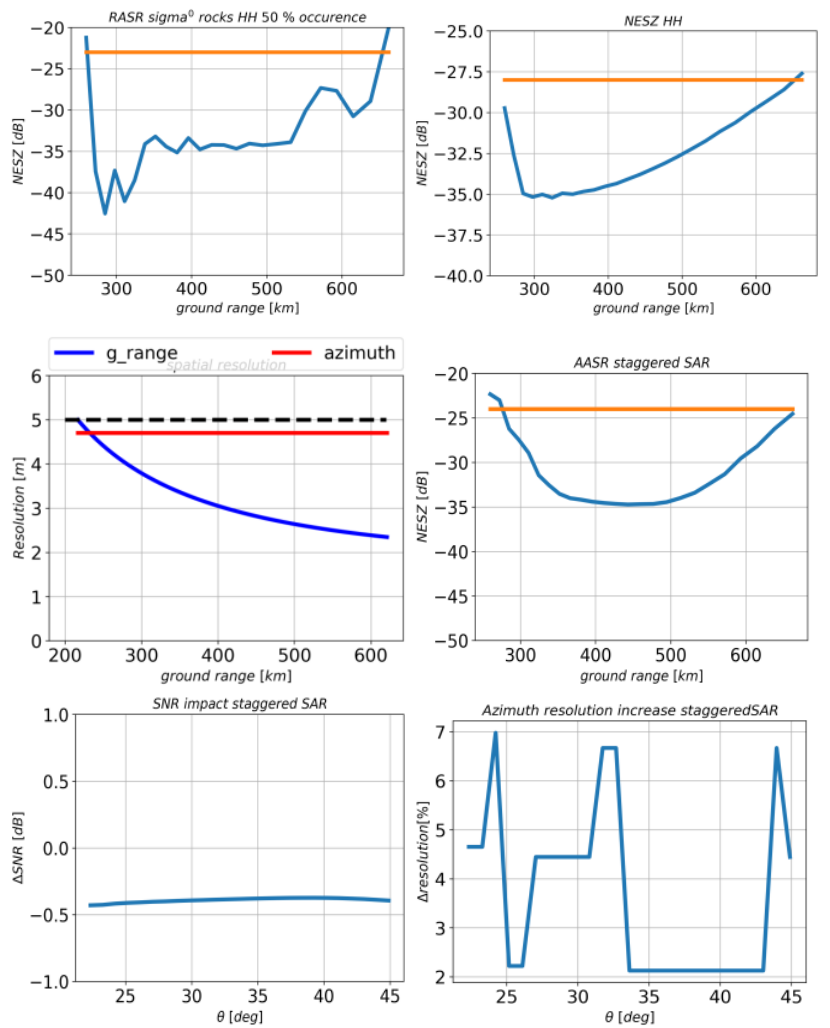

Fig. 10. SAR derived performances of the baseline antenna concept.

\section{CONCLUSION}

In this paper, a L-band multi-channel feedarray reflector antenna based SAR system concept has been presented as suitable architecture for next generation HRWS SAR missions based on digital beam forming and advanced operation modes (i.e. SCORE and Staggerd PRI). Different beamforming concepts, antenna architectures and feedarray implementation techniques have been discussed. The proposed SAR system concept is capable to map $400 \mathrm{~km}$ swath with 5 meter resolution, achieving NESZ better than $28 \mathrm{~dB}$ and TAR lower than $-22 \mathrm{~dB}$.

\section{REFERENCES}

[1] G. Krieger, N. Gebert, M. Younis, F. Bordoni, A. Patyuchenko, A. Moreira, "Advanced concepts for ultra-wide swath SAR imaging," in Proc. European Conf. Synthetic Aperture Radar, Friedrichshafen, Germany, June 2008

[2] M. Younis, C. Fischer, W. Wiesbeck, "Digital beamforming in SAR systems," IEEE Trans. Geosci. Rem. Sens., Vol. 41, 2003

[3] N. Gebert, G. Krieger, and A. Moreira. "Digital beamforming on receive: Techniques and optimization strategies for high resolution wide-swath SAR imaging," IEEE Transactions on Aerospace and Electronic Systems, Vol. 45, pp. 564-592, 200

[4] G. Krieger, F. Queiroz de Almeida, S. Huber, M. Villano, M. Younis, A. Moreira, J. Del Castillo, M. Rodriguez Cassola, P. Prats, D. Petrolati, M. Ludwig, C. Buck, M. Suess, N. Gebert, "Advanced LBand SAR System Concepts for High-Resolution Ultra-Wide-Swath SAR Imaging", ESA Advanced RF Sensors and Remote Sensing Instruments (ARSI), 12-14 September 2017 ESA ESTEC, Noordwijk, The Netherlands.

[5] S. Huber, M. Younis, and G. Krieger "Tandem-L: SAR System Design Aspects". 5th Workshop on Advanced RF Sensors and
Remote Sensing Instruments, ARSI'17 \& 3rd Ka-band Earth Observation Radar Missions Workshop, KEO'17

[6] M. Villano, G. Krieger, Marc Jäger, A. Moreira,"Staggered SAR Performance Analysis and experiments with real data". IEEE Transactions on Geoscience and remote sensing, Vol 55, $\mathrm{n}^{\circ} 11$, November 2017

[7] F. Queiroz de Almeida, M. Younis, G. Krieger, A. Moreira "Multichannel Staggered SAR Azimuth Processing", IEEE Transactions on Geoscience and remote sensing, Vol 56, $\mathrm{n}^{\circ} 5$, May 2018

[8] D. Betancourt-Quiñones, A. Ibánez-Loinaz, R. García-Esparza, and C. Del-Río-Bocio, "Designing antenna systems with CORPS (Coherently Radiating Periodic Structures)," in 28th ESA Antenna Technology Workshop on Space Antenna Systems and Technologies, 2005, pp. 509-516.

[9] D. Betancourt and C. del Río Bocio, "A novel methodology to feed phased array antennas," IEEE Trans. Antennas Propag., vol. 55, no. 9, pp. 2489-2494, 2007, doi: 10.1109/TAP.2007.904133.

[10] U. H. Gysel, "A New N-Way Power Divider/Combiner Suitable for High-Power Applications," Mar. 1975, pp. 116-118, doi: 10.1109/mwsym.1975.1123301.

[11] N.Llombart, A.Neto, G.Gerini, M.Bonnedal, P.De Maagt, "Leaky Wave Enhanced Feed Arrays for the Improvement of the Edge of Coverage Gain in Multibeam Reflector Antennas", IEEE Transactions on Antennas and Propagation, Vol. 56, No. 5, pp. 1280-1291, May 2008

[12] F.T. Ulaby and M. Dobson, Handbook of Radar Scattering Statistics for Terrain ,Artech House, Inc., 1989 